\title{
CSF Electrophoresis in One Thousand Patients
}

\author{
G.C. EBERS and D.W. PATY
}

SUMMARY: Agarose and/or cellulose acetate electrophoresis was performed on the CSF of one thousand patients. In patients with clinically definite multiple sclerosis $(M S)(N=267) 92.8 \%$ had oligoclonal banding (O.B.). In patients with possible $M S(N=283) O . B$. was present in $31.1 \%$. In patients with other neurological diseases $(N=450) O . B$. was present in $8 \%(N=36)$. Nineteen non- $M S$ patients with positive $O . B$. had serum

RESUME: Chez mille patients nous avons étudié le LCR par électrophorèse sur agarose et/ou acetate de cellulose. Chez les patients avec sclérose en plaques cliniquement certaine $(N=267), 92.8 \%$ ont une bande oligoclonale (O. B.). Chez les patients avec SEP possible $(N=283)$, O.B. fut trouvée dans $31.1 \%$ des cas. En ce qui concerne les patients avec autres maladies neurologiques $(N=450), 8 \%(N=36)$ avaient une bande oligoclonale. 19 patients, bands or disorders known to be associated with local immune response. The remaining 17 patients had no explanation for the oligoclonal banding. In the majority of these MS had not been a diagnostic consideration.

CSF electrophoresis is the single most reliable laboratory test in multiple sclerosis and deserves incorporation into the diagnostic criteria for the disease.

non $S E P$, avec $O . B$. positive avaient des bandes sériques ou des désordres connus comme de nature immunitaire locale. Aucune explication satisfaisante de l'O.B. ne fut trouvée chez les autres 17 patients, pour la majorité desquels la SEP ne fut pas une considération diagnostique.

L'électrophorèse du LCR est le test de laboratoire le plus fiable dans la SEP et devrait être incorporé aux critères diagnostiques de la maladie.
From the Department of Clinical Neurological Sciences, University Hospital, University of Western Ontario, London, Ontario, Canada.

Reprint requests to Dr. G.C. Ebers, Division of Neurology, University Hospital, 339 Windermere Road, London, Ontario N6A 5A5 Canada.

\section{INTRODUCTION}

The diagnostic detection of immunological abnormalities in the cerebrospinal fluid (CSF) of patients with multiple sclerosis (MS) originated in 1947 when Kabat (1942) demonstrated the elevated IgG levels often seen in the disorder. Since then a number of additional observations have extended the initial findings. The introduction of a simple technique of agar electrophoresis (Wieme, 1959) led to the observation that the IgG elevation was electrophoretically restricted (oligoclonal banding - OB) (Lowenthal et al., 1960). There is some reason to think that the immune response exemplified by $O B$ is a specific one. The antibody response seen in the CSF shows electrophoretic restriction, an altered kappa-lambda light chain ratio (Link et al., 1970) and IgG subclass restriction (Vandvik et al., 1976). Although the nature of the antibody response remains uncertain, its presence is of diagnostic usefulness in the evaluation of patients with multiple sclerosis and other inflammatory neurological diseases.

In spite of much evidence that CSF electrophoresis is valuable in the diagnosis of MS, neurological centers in this country have been slow to adopt this methodology. The present paper details the experience with CSF electrophoresis in the Multiple Sclerosis Clinic at University Hospital, London, Ontario, for the last 4 years. The diagnostic value of CSF electrophoresis shown by other workers is confirmed.

PATIENT MATERIAL

CSF samples submitted to the University Hospital Biochemistry Laboratory for electrophoresis were provided largely by members of the Department of Clinical Neurological Sciences. Additional CSF samples were submitted from other hospitals in 
TABLE 1

Results of CSF Electrophoresis for Oligiclonal Banding in 1,000 Patients (Combined Results of Cellulose Acetate and Agarose)
1000 CSF ELECTROPHORESES USING CELLULOSE ACETATE AND AGAROSE
O.B. NEG \% POS

248

108

36
19

175

414
92.8\% C.D.M.S.

$38.1 \%$

$8.0 \%$
POSS MS

NON MS
O.B. POS

O.B. NEG

$\%$ POS

\section{${ }^{*}$ C.D.M.S. - clinically definite MS}

the area. More than $90 \%$ of the patients with clinically definite MS (C.D.M.S.) were examined by one of the authors. In addition to CSF submitted for diagnosis, many patients with well documented MS agreed to lumbar punctures for confirmation of the diagnosis as part of a research protocol.

Patients were divided into groups of "C.D.M.S.", "possible MS" (which included Rose's possible and probable MS) - (Rose et al., 1976) and "nonMS". Possible MS was loosely interpred as including cases in which MS was a diagnostic possibility although other diagnoses were under consideration and in many instances more likely. Some patients known to the authors with a clinical status of "possible MS" at the time of CSF electrophoresis, but who subsequently fulfilled criteria for C.D.M.S. during the period of the study were included in the C.D.M.S. group. (This small group of patients is presently under study to determine the prognostic usefulness of CSF electrophoresis in possible MS).

\section{METHODS}

Cerebrospinal fluid was obtained by lumbar puncture and after centrifugation was concentrated using Minicon B15 filters (Amicon Corporation, Lexington, Mass.), or by negative pressure dialysis. CSF protein was determined by TCA precipitation (Meulemans, 1960) and samples were concentrated to between 40 and 100 times the original volume so that the total protein of the concentrated CSF approximated 2-3 gm\%. The first 650 samples were done by cellulose acetate electrophoresis coupled with a photographic enhancement technique as previously described (Paty et al., 1977). The last 350 samples were done by agarose gel electrophoresis using either a commercial electrophoresis system (Panagel - Millipore Corporation, Bedford, Mass.) or with agarose plates prepared in the author's lab using high electroendosmotic agarose (Seakem - Marine Colloids, Rockland, ME). The method for running electrophoresis, drying gels and staining is as previously described (Johnson et al., 1977) (LKB application note \#310 - LKB, Bromma, Sweden). Gels were evaluated visually for the presence of oligoclonal banding. This was done without knowledge of the diagnosis except in the case of the authors' own patients. Gels were considered positive if two or more bands were present in the gammaglobulin region excluding those commonly found in control CSF (gamma trace protein) (Link, 1967) and (with agarose) a band between the origin and gamma trace protein (Laurell, 1972). For some studies an anti-IgG overlay technique (Arnaud et al., 1977) was used for immunochemical confirmation of the immunoglobulin nature of bands. Isoelectric focusing was performed using gels made in the authors' laboratory immediately prior to use (LKB application note \#75, Ebers et al., 1979).

$\begin{array}{rrr}103 & 5 & 95.4 \% \\ 35 & 53 & 39.8 \% \\ 11 & 143 & 7.1 \%\end{array}$

Electrophoreses which were technically inadequate were not included in the series. This usually related to insufficient CSF being supplied or to unusually low protein levels.

\section{RESULTS}

Our results confirm previous findings that CSF electrophoresis in C.D.M.S. is of high diagnostic yield (Laterre et al., 1970). It can be seen from the overall results of cellulose acetate and agarose gel electrophoresis that oligoclonal banding was found in $92.8 \%$ of 248 patients with C.D.M.S. (Table 1). Agarose electrophoresis alone yielded 95.4\% positive O.B. in 103 patients (Table 2). The incidence of O.B. in "possible MS" was substantially lower than that in C.D.M.S. (38.1\% of 283 patients) in the combined series.

The variety of diagnoses seen in patients with negative O.B. is in Tables 3 and 4 . These largely reflect a cross section of patients undergoing lumbar puncture or myelography. Table 5 lists the diagnoses in patients having oligoclonal banding in the CSF with neurological disorders other than MS. We have divided these 36 patients into two groups, one associated with local immune response or serum bands, and the other not associated with either of these two features. Of the 36 non-MS patients with O.B. 19 fell into the former group and the presence of O.B. in CSF appeared to reflect a local CNS immune response or passive diffusion of bands from the serum. For the remaining 17 patients we have no explanation for the presence of O.B. 
TABLE 3

Diagnoses in 414 Patients With Negative Oligoclonal Banding

CSF NEGATIVE FOR OLIGOCLONAL BANDING NON-MS NEUROLOGIC DISORDERS $(\mathrm{N}=414)$

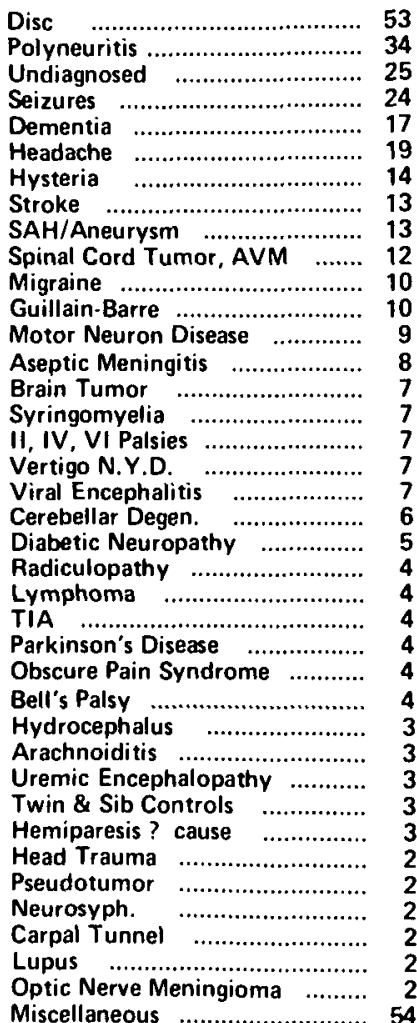

TABLE 4

Miscellaneous Patients With Negative CSF Electrophoresis for Oligoclonal Banding

MISCELLANEOUS PATIENTS WITH NEGATIVE O.B. (N $=54)$

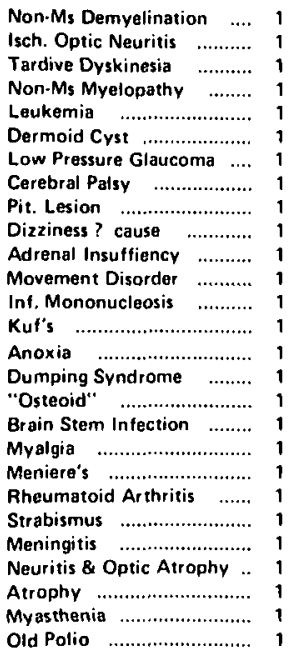

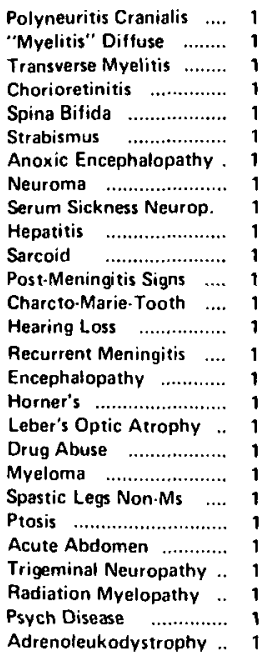

The results with agarose were slightly better than those with cellulose acetate reflecting a slight increase in sensitivity with this method. As previously reported (Ebers et al., 1979) isoelectric focusing, agarose gel electrophoresis and cellulose acetate electrophoresis all gave high yields in C.D.M.S. in decreasing order of sensitivity. Agarose electrophoresis in $6 \mathrm{MS} \mathrm{CSF}$, one control CSF and normal serum is seen in the Figure.

\section{DISCUSSION}

Data obtained in this study are comparable to those obtained by Olsson et al. (1979) with agarose electrophoresis. The incidence of O.B. in "possible MS" was substantially lower than in C.D.M.S. As noted above this partly reflects the liberal interpretation of possible MS. On the other hand, a substantial number of patients in this group with clinically monosymptomatic demyelination were included in the positive, and to a much lesser extent in the negative O.B. group.

Studies are underway to document the prognostic significance of O.B. in monosymptomatic patients with respect to future dissemination. Further, the frequency of O.B. developing in the course of the disease, having been absent earlier, is still an open question. This development is suggested by the lower frequency of O.B. in the "possible" group. The development of bands has been claimed in optic neuritis progressing to MS, (SandbergWolheim, 1975). Of relevance in this regard is the fact that the banding pattern once present in MS tends to remain stable (Vandvik, 1977).

Our own observations on this point have been hampered by improving the methodology over the course of time which has made it difficult to exclude the possibility that prior absence of O.B. was simply due to technical factors.

The appearance of O.B. in disorders other than MS clearly demonstrates that the presence of oligoclonal banding in CSF is not diagnostic of MS. The phenomenon of oligoclonal banding (which appears to represent the activation of relatively few clones of immunoglobulin producing cells) may be seen in a variety of disorders in addition to MS. These disorders are in general unlikely to cause diagnostic confusion. In a single case in this series a patient with the Arnold-Chiari malformation was thought to have clinical MS partly on the basis of a suspicious CSF electrophoresis, but follow-up led to the correct diagnosis.

The importance of concomitant serum paraprotein abnormalities were reflected in the CSF. We have not done the serum electrophoresis routinely until the most recent 350 samples.

Seventeen patients with "non-MS" O.B. are difficult to explain. We have observed the persistence of O.B. in an occasional patient with herpes simplex encephalitis and a similar phenomenon has previously been reported for mumps meningo-encephalitis (Vandvik et al, 1978). It is possible that bands in these patients could have represented the residua of a remote viral infection of the nervous system. It seems an unlikely explanation however, for the majority of these non-MS positives.

Because immunofixation was not routinely used in this study some oligoclonal bands may not have been immunoglobulin and accordingly it is possible that routine use of an anti-lgG overlay method might reduce the frequency of non-MS positives. However, this remains to be seen.

Recently we have employed an antiIgG peroxidase overlay method to determine that certain bands are immunoglobulin, but this was not routinely used. The results given in Tables 1 through 5 were obtained without this modification. Because of the slightly better results of the agarose method and the lack of necessity for photographic enhancement our laboratory now uses the agarose method as a routine.

The data from this large series confirms information gathered by numerous individuals on the diagnostic value of CSF electrophoresis in MS. The frequency of O.B. in this study is very high although similar results have been reported (Olsson et al., 1976; Laurenzi et al., 1978). We have been concerned that the high frequency of O.B. in our study reflects to some extent the large number of well developed cases included in this series. There is a paucity of data on how often O.B. develops in the course of MS to 
indicate how important the duration of the disease is to the frequency of O.B.

Nevertheless, the frequency of abnormalities has been so high as to substantially affect our clinical practice. The presence of O.B. in CSF has frequently led to the omission of contrast procedures such as myelography which would ordinarily have been done. Furthermore, the absence of O.B. when it is expected has occasionally led to the identification of another non-MS diagnosis. We think it in order to reconsider the diagnostic criteria for MS.

Heretofore, the accepted diagnostic criteria for MS (Schumacher et al., 1965) have been solely clinical. The accuracy of these clinical diagnostic criteria has never been established. There is no question that numerous individuals may fulfill the diagnostic criteria for MS and at autopsy be proven to have another disorder.
Heavy reliance on subjective interpretations are implicit in the criteria, especially in the caveat that the disease cannot be better explained by another disorder. In the absence of careful autopsy data arguments on the relative merits of clinical criteria and CSF electrophoresis become circular. For research studies on MS we favor the view that only patients with O.B. be included. It is worth pointing out that no autopsy study evaluating the reliability of the clinical criteria has been performed or is likely to be in the near future. Our own material included 9 autopsy proven cases of MS seen during the last 4 years. All of these patients have shown O.B. in life or in electrophoresis of post-mortem CSF. However, an additional autopsied patient with "transitional sclerosis" did not have oligoclonal banding. One autopsy case of "clinical MS" was found to have a neuro-enteric cyst embedded in the thoracic cord. There was no evidence of multiple sclerosis. In this case O.B. was negative on postmortem CSF obtained at cisternal puncture.

We have been interested to determine if the results of agarose gel electrophoresis can be improved with the use of isoelectric focusing (IEF). IEF is a more sensitive technique than agarose gel and frequently separates bands, which are apparently single on agarose, into several distinct ones (Ebers et al., 1980). It has been shown that single antibodies secreted by cloned myeloma cells may be resolved into a number of bands probably reflecting post-synthetic differences among antibody molecules. We have found IEF especially useful for those cases in which agarose electrophoresis was equivocal or negative as suggested by Laurenzi (1978). No case has been found in which the agarose gel electrophoresis was positive and the IEF negative. Interestingly, the cases in which IEF was positive

\section{TABLE 5}

Diagnoses in Non-MS Patients Positive for Oligoclonal Banding

NON-MS - POSITIVE FOR OLIGOCLONAL BANDING

Disorders Associated with Local Immune Response or Serum Bands

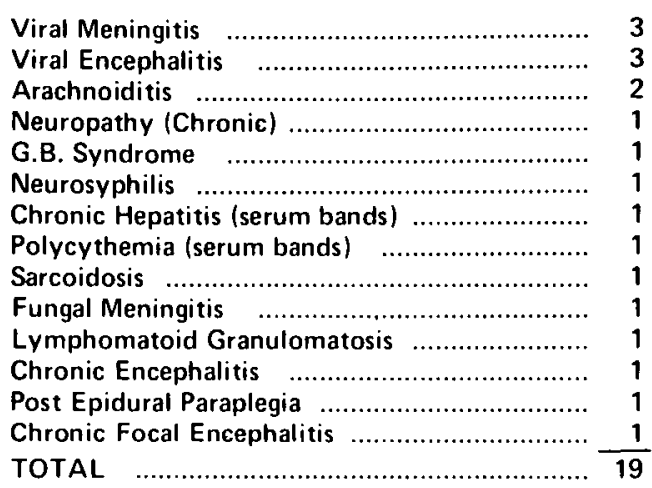

NON.MS - POSITIVE FOR OLIGOCLONAL BANDING

Disorders Not Associated with Local Immune Response or Serum Bands

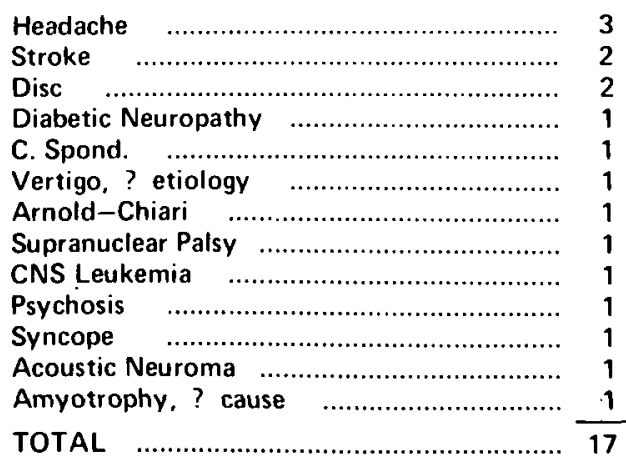


and agarose was negative were those in which there were a substantial number of IgG bands of roughly the same density so that the agarose electrophoresis appeared to give a homogeneous IgG population. Accordingly, with IEF we found that approximately half of the patients with C.D.M.S. not having banding by agarose were shown to have O.B. This then leaves perhaps $2-3 \%$ of our patients with C.D.M.S. who do not have oligoclonal banding by any of the three methods.

The demonstration of O.B. in patients with C.D.M.S. is usually not of great practical value, although we think it increases the diagnostic certainty. As mentioned above, its absence has led to alternative diagnoses. The demonstration of O.B. is of greater importance in those cases where there is clinical uncertainty and may favor the diagnosis of MS when this is not possible using clinical criteria. Prolonged follow-up will be required to determine the frequency of O.B. in cases of "possible MS" which progress to C.D.M.S.

\section{TECHNICAL COMMENTS}

The method of concentration of CSF used in this study was adequate. In a previous study concentration of CSF was varied so that a constant amount of immunoglobulin per cent was applied to the gel (Link, 1973). Since the IgG level in MS CSF is often much higher than that seen in controls, this approach may require a very high concentration of non-MS CSF or less concentration of MS CSF. We have not found this an advantage. We now use the quantity of albumin (an index of barrier function) as a standard for protein applied to gels. Albumin and IgG levels may be determined simultaneously by rocket immunoelectrophoresis as described by Tourtellotte (1969). Since nephelometric determinations of IgG and albumin levels may be performed in many routine hospital laboratories, rapid measurement of $\mathrm{lgG}$ and albumin may be obtained with this method. Nevertheless, as shown in this study adequate results can be obtained simply with total protein.

\section{CONDUCTION MEDIUM}

Some variability has been noted in the commercial agarose plates which leads to occasional difficulties. However, on the whole the commercial apparatus and gels have been adequate. The main advantages of the commercial system relate to the narrow application points enabled by the slotted masks and the fact that the agarose is bound to a plastic backing for ease of handling.

For gels poured in our own lab we have used high electroendosmotic agarose. This increases cathodal migration of $\mathrm{lgG}$ somewhat and may aid in the separation of oligoclonal bands.

We have found it important to run normal serum and CSF samples with each electrophoresis as standards. Paired sera and CSF's should be run simultaneously to identify CSF bands positively originating from the serum compartment.

\section{CONCLUSIONS}

The demonstration of oligoclonal banding by CSF electrophoresis is of substantial diagnostic value in the evaluation of MS. Good results may be obtained using easily adaptable commercial electrophoresis systems. These are within the capability of the average hospital biochemistry laboratory. In this study $92.8 \%$ of patients with clinically definite MS had oligoclonal banding. Eight percent of non-MS neurological disorders also demonstrated oligoclonal banding. The majority of these disorders were those associated with local CNS immune response or serum bands. The CSF electrophoresis appears to have a sufficient diagnostic yield to warrant inclusion in the criteria for the diagnosis of MS.

NOTE: This project was funded by a grant from the Physicians" Services Incorporated Foundation.

ADDENDUM: C.D.M.S. patients in this series had a mean duration of disease at the time of CSF study of 11 years. (median 7 years)

\section{REFERENCES}

ARNAUD, P., WILSON, G.D., KOISTINEN, J. and FUDENBERG, H. (1977). Immunofixation after electrofocusing; improved method for specific detection of serum proteins with determination of isoelectric points. J. Immunol. Methods 16, 221-231.

EBERS, G.C. and PATY, D.W. (1979). Alkaline isoelectric focusing in multiple sclerosis. Trans. Am. Neurol. Ass. (in press).

EBERS, G.C., RICE, G.P. and ARMSTRONG, $H$. (1980) Preparative Isoelectric Focusing in Agarose. J. Immunol. Methods (in press).

JOHNSON, K.P., ARRIGO, S.C., NELSON, B.J. and GINSBERG, A. (1977). Agarose electrophoresis of cerebrospinal fluid in multiple sclerosis. Neurology 27, 273-277.
KABAT, E.A.. MOORE, D. H. and LANDOW H. (1942). An electrophoretic study of the protein components in the cerebrospinal fluid and their relationship to serum proteins. J. Clin. Invest. 21, 571-577.

LATERRE, E.C., CALlEWAERT, A. and HEREMENS, Y.S. (1970). Electrophoretic morphology of gammaglobulins in cerebrospinal fluid of multiple sclerosis and other disease of the nervous system. Neurology 20. 982-990.

LAURELL, C.B. (1972). Composition and variation of the gel electrophoretic fractions of plasma, cerebrospinal fluid and urine. Scand. J. Clin. Lab. Invest. 29, (suppl. 124) $71-82$.

LAURENZI, M.A. and LINK, H. (1978). Comparison between agarose gel electrophoresis and isoelectric focusing of CSF for demonstration of oligoclonal immunoglobulin bands in neurological disorders. Acta Neurol. Scandinav. 15, 148-156.

LINK, H. and ZETTERVALL, O. (1970) Multiple sclerosis - disturbed kappa: lambda light chain ratio of immunoglobulin $G$ in the cerebrospinal fluid. Clin. Exp. Immunol. 6, 435-438.

LINK, H. (1973). Comparison of electrophoresis on agarogel and agarose gel in the evaluation of gamma globulin abnormalities in cerebrospinal fluid in the serum in multiple sclerosis. Clin. Chim. Acta 46, 383-389.

LOWENTHAL, A., VANSANDE, M. and KARCHER, D. (1960). The differential diagnosis of neurological disease by fractionating electrophoretically the CSF-globulins. J. Neurochem. 6, 51 .

MEULEMANS, O. (1960). Determination of total protein in spinal fluid with sulphosalicylic and trichloracetic acid. Clinica chim. Acta $5,757$.

OLLSON, J.E., LINK, H. and MULLER, R. (1976). Immunoglobulin abnormalities in multiple sclerosis. J. Neurol. Sci. 27, 233245.

PATY, D.W., DONNELLY, M. and BERNARDO, M.E. (1978). CSF electrophoresis: An adaptation using cellulose acetate for the identification of oligoclonal banding. Can. J. Neurol. Sci. 5. 297-299.

ROSE, A.S., ELLISON, G., MYERS, L.. et al. (1976). Criteria for the clinical diagnosis of multiple sclerosis. Neurology 26, 20-22.

SCHUMACHER, G.A., BEEBE, G., KIBLER, R.F., KURLAND, L.T., KURTZKE, J., MCDOWELL, F., NAGLER, B., SIBLEY, W.A., TOURTELLOTTE, W.W. and WILLMON, T.L. (1965). Problems of experimental trials of therapy in multiple sclerosis: Report by the panel of the evaluation of experimental trials of therapy in multiple sclerosis. Ann. N.Y. Acad. Sci. $122,552-568$. 
TOURTELLOTTE, W.W., TAVOLATO, B., PARKER, J.A. and COMISO, P. (197I). Cerebrospinal fluid electroimmunodiffusion in multiple sclerosis and other neurological diseases. Arch. Neurol. 25, 345-350.

VANDVIK, B., NATVIG, J.B. and NIGER, D. (1976). IgGl subclass restriction of oligoclonal $\operatorname{lgG}$ from cerebrospinal fluids and brain extracts in patients with multiple sclerosis and subacute encephalitides. Scand. J. Immunol. 5, 427-436.

VANDVIK, B. (1977). Oligoclonal IgG and free lightchains in the cerebrospinal fluid of patients with multiple sclerosis and infectious diseases of the central nervous system. Scand. J. Immunol. 6, 913-922.
VANDVIK, B., NORRBY, E., STEENJOHNSEN, J. and STENSVOLD, $K$. (1978). Mump meningitis - prolonged pleocytosis and occurrence of mumps virus specific oligoclonal IgG in the CSF. Europ. Neurol. 17, 13-22.

WEIME, R.J. (1959). Studies on agar gel electrophoresis. Clin. Chim. Acta 4, 317-321. 